\title{
REZENSIONEN
}

\section{Nationale Parlamente in der EU-Rechtsetzung: problematische oder positive Einbindung?}

Janowski, Cordula Agnes: Die nationalen Parlamente und ihre Europa-Gremien. Legitimationsgarant der EU?, Nomos Verlagsgesellschaft, Baden-Baden 2005, 278 Seiten, € 49,-.

Mellein, Christine: Subsidiaritätskontrolle durch nationale Parlamente. Eine Untersuchung zur Rolle der mitgliedstaatlichen Parlamente in der Architektur Europas, Nomos Verlagsgesellschaft, Baden-Baden 2007, 527 Seiten, € 89,-.

Kaum ein anderes Element des hoffentlich nur vorerst gescheiterten Vertrags über eine Verfassung für Europa (VVE) ist in der öffentlichen Diskussion so begrüßt, aber gleichzeitig auch so wenig kritisch hinterfragt worden wie die von ihm intendierte Rolle der nationalen Parlamente in der Europäischen Union, insbesondere bei der Sicherung des Prinzips der Subsidiarität. Diese Kriterien näher zu prüfen ist auch nach der Ablösung des VVE durch den Vertrag von Lissabon ${ }^{1}$ zur Änderung der Verträge über die EU und zur Gründung der EG (VL) relevant geblieben, da der VL die dem VVE angefügten Protokolle über die Rolle der nationalen Parlamente in der EU und über die Anwendung der Grundsätze der Subsidiarität und der Verhältnismäßigkeit wörtlich übernimmt, die Anwendungsfälle im Subsidiaritätsprotokoll sogar noch um eine Variante ${ }^{2}$ erweitert und überdies der Rolle der nationalen Parlamente nunmehr sogar einen eigenen Vertragsartikel ${ }^{3}$ widmet. Um so mehr am Platze sind deshalb nach wie vor zwei wissenschaftliche Untersuchungen auf der Grundlage jener Kriterien, auch wenn sie zu verschiedenen Ergebnissen kommen.

Um das Ergebnis eines Gesamtvergleichs der beiden Monographien und ihrer wesentlichen Inhalte vorwegzunehmen: Die Untersuchung von Cordula Janowski zeichnet sich dadurch aus, dass sie aus verfassungsrechtlicher Sicht zu einer sehr kritischen Beurteilung der Rolle der nationalen Parlamente in den EU-Strukturen kommt und auch ihre praktische Effizienz in den mitgliedstaatlichen Systemen eher gering einschätzt. Demgegenüber bewertet Christine Mellein die den nationalen Parlamenten auf europäischer Ebene zugedachten Funktionen als rechtlich überaus positiv und bedenkenfrei.

Im Einzelnen hebt Janowski mit Recht hervor, dass die nationalen Parlamente als Gegenstände der Integrationsforschung weitgehend erst in jüngster Zeit „entdeckt“ worden seien. Erst recht gelte das für eine ihnen zugeschriebene eigene „europäische Legitimationsfunktion“, die sich jedenfalls in der deutschen Debatte wesentlich aus dem Maastricht-Urteil des Bundesverfassungsgerichts herleitet. Das Gericht hatte den Beitrag der nationalen Par-

1 Text siehe BR-Drucks. 928/07.

2 Art. 7 Abs. 3.

3 Art. 8c VL. 
lamente neben dem des Europäischen Parlaments als „unabdingbar“ zur Vermeidung einer demokratietheoretischen Krise angesehen und so zu dem heute der herrschenden Meinung entsprechenden dualen Legitimationsmodell geführt (S. 38 - 41). Trotz jener Stützung durch besagte herrschende Meinung wird man diesem Begriff - wie manch anderem aus dem Maastricht-Urteil - wohl einige Fragezeichen entgegenstellen müssen. Janowskis Schrift kommt vor allem das Verdienst zu, dies in zwei wichtigen Bereichen nachhaltig unternommen zu haben: Sie hinterfragt die praktische Ausgestaltung innerhalb der nationalen Parlamente und die Einpassung ihres so genannten Frühwarnsystems zum Subsidiaritätsprinzip in die EU-Strukturen in deren Verhältnis zu den Verfassungssystemen der Mitgliedstaaten.

Janowski untersucht den EU-Ausschuss des Deutschen Bundestages in vergleichender Perspektive mit den Europa-Gremien der Abgeordnetenkammern in den Parlamenten Österreichs, Dänemarks, Schwedens, Finnlands, Frankreichs, Großbritanniens, Irlands, Belgiens, der Niederlande, Luxemburgs, Italiens, Spaniens, Portugals und Griechenlands (S. 69 - 184). Daran ist allein zu bedauern, dass die Nichteinbeziehung der so genannten Zweiten Kammern gerade in diesem Bereich einige wichtige Erkenntnisse zur Arbeitsweise auch der Abgeordnetenkammern nicht zur Geltung kommen lässt. Zumindest gilt das für den Bundestag im Verhältnis zum Bundesrat, dessen Vorarbeiten für Stellungnahmen zu EU-Vorhaben im eigenen EU-Ausschuss in aller Regel die Basis für die Beratungen des Bundestagsausschusses bilden und in dem die Willensbildung ohne diese Grundlagen erheblich erschwert wäre. Die vergleichende Analyse Janowskis ergibt auch in dieser Begrenzung „ein ausgesprochen ernüchterndes Bild für die ... zugrunde liegende Fragestellung“ nach Berechtigung und Realität des dualen Legitimationsmodells (S. 178). Konkret resümiert die Verfasserin, „die Tatsache, dass zehn der ... untersuchten 15 nationalen Parlamente nicht über eine hinreichende Basis für eine europapolitische Mitwirkung ... verfügen, (werfe) Zweifel an der universellen Geltungskraft“ des dualen Modells auf und stelle es „somit auch ... grundsätzlich infrage" (S. 183).

Nach einer sehr übersichtlichen tabellarischen Aufbereitung ihrer Vergleichsergebnisse (S. 184) unterzieht die Verfasserin die „kompetenzstarken Europa-Gremien“ auch einem Praxistest in vier Feldern: Betrugsbekämpfung und Grundrechtekonvent (beide untersucht am EU-Ausschuss des Bundestages), Tiertransporte (EU-Hauptausschuss des österreichischen Nationalrats) und Einflussnahmen der so genannten COSAC (Konferenz der Ausschüsse für Gemeinschafts- und Europa-Angelegenheiten der Parlamente der Europäischen Union) auf den Verfassungskonvent (S. 192 - 226). Im Ergebnis steht „ein fast ebenso ernüchterndes Bild“ wie das zu der vorangegangenen Analyse (S. 221). Janowski wirft deshalb mit Recht die Frage auf, „welche Europa-Gremien überhaupt die vom dualen Legitimationsmodell angenommene Rückkopplung der EU und ihrer Maßnahmen zu den Parlamenten in der Verfassungswirklichkeit herstellen“, und sie folgert, „dass es zur Umsetzung der Theorie in die Praxis bisher nur vereinzelt gekommen ist" (S. 226).

Bliebe dies der einzige Kritikpunkt am so genannten Frühwarnsystem, so könnte man sich noch mit dem Hinweis und vielleicht der Aussicht trösten, dass diese Insuffizienzen nicht so bleiben müssten und sich mit steigendem europäischen Problembewusstsein der nationalen Parlamente in der Praxis jenes Systems auch beheben ließen. Nur ist der dargestellte empirische Befund eben nicht der einzige Kritikpunkt. Gewichtiger, weil eben nicht überwindbar, ist ein verfassungssystematischer: Mit den für das Frühwarnsystem zu schaffenden Institutionen und Verfahren kommt es zu einer Vermischung der Verfassungsstruk- 
turen von Union und Mitgliedstaaten. Dadurch werden die nationalen Parlamente ohne förmliche Organstellung in eine europäische Verantwortlichkeit genommen, durch deren Wahrnehmung sie gleichzeitig daran gehindert werden können, ihren primären nationalen Verpflichtungen im EU-Bereich nachzukommen - nämlich ihre Regierungen für deren Verhalten im Rat zur Verantwortung zu ziehen. Einwendungen gegen eine EU-Vorlage aus dem nationalen Bereich aber gehören in den Rat als der Kammer der Mitgliedstaaten und nicht in eine amorphe Summe von Voten der Parlamente im Frühwarnsystem. Wie also will sich ein nationales Parlament verhalten, wenn die eigene Regierung im Rat sich bereits für eine bestimmte Vorlage ausgesprochen hat oder - auch aus Mehrheitsgründen - aussprechen will oder muss, während das Parlament im Frühwarnsystem gegenteilig votieren will oder schon votiert hat? Es bleibt ihm dann entweder nur eine Rüge gegen die eigene Regierung oder eine Aufgabe oder Aufhebung des eigenen Votums - eine Situation, in der die Folgen jener Vermischung von EU- und nationalen Verfassungsstrukturen sehr deutlich zu Tage treten.

Janowski hat diese Folgen zwar nicht expressis verbis zum Ausdruck gebracht. Sie hat aber mit Recht darauf hingewiesen, dass mit dem Frühwarnsystem „de facto ein Drei-Kammer-Verfahren“ geschaffen werde, mit dem den nationalen Parlamenten „die Möglichkeit (eröffnet werde), an dem Organ vorbei zu handeln, dem der Schutz der nationalen Kompetenzen ... obliegt - dem Rat“ (S. 231). Ihre Folgerung lässt an Klarheit nichts vermissen; es bleibt nur zu wünschen, dass sie vor einem realen Einstieg in das Frühwarnsystem auch Beachtung findet: „Die direkte Einbindung der nationalen Parlamente in die EU-Rechtsetzung ist ... systemwidrig, denn die nationalen Parlamente sind kein Unionsorgan und sollen es auch nicht werden" (ebenda).

Christine Mellein kommt demgegenüber in geradezu euphorischer Argumentation zu dem Ergebnis, „die Stärkung des prozeduralen Aspekts des Subsidiaritätsprinzips unter Einbeziehung der nationalen Parlamente (sei) ... (zu) begrüßen“ (S. 490). Gemäß ihren eigenen Zielsetzungen konzentriert sich die Verfasserin „auf die spezifische Ausgestaltung und den Umfang (der) neuen Mechanismen, ihre Sachdienlichkeit im Rahmen des EU-Systems und ihre potenziellen Auswirkungen auf das institutionelle Gefüge der EU wie auch auf die mitgliedstaatlichen Verfassungsordnungen“ (S. 32).

Melleins Buch ist in vier Teile gegliedert: Zunächst wird in einer Bestandsaufnahme die „bisherige Rolle der nationalen Parlamente in der Architektur Europas“ geschildert. Danach wird die „Stellung der nationalen Parlamente nach dem Europäischen Verfassungsvertrag“ dargestellt. Dem folgt ein dritter Teil über „Mögliche Auswirkungen der Subsidiaritätskontrollmechanismen auf die mitgliedstaatlichen Verfassungssysteme - eine exemplarische Untersuchung der Grundstrukturen der Beteiligung nationaler Parlamente in Deutschland und Großbritannien“, von dem man eigentlich die entscheidenden Ausführungen zu den bereits skizzierten Konfliktkonstellationen erwarten müsste. Die Schrift schließt mit „Perspektiven der Subsidiaritätskontrolle durch nationale Parlamente“, die eine Zusammenfassung der Ergebnisse einbeziehen.

Positiv hervorzuheben ist, dass die Publikation auf der Grundlage eines sorgfältigen Vergleichs der sehr unterschiedlichen Mechanismen zur Behandlung von EU-Angelegenheiten in Deutschland und Großbritannien (in dessen Parlament der Hauptanteil dabei dem House of Lords zufällt) zahlreiche konkrete „Eckpunkte“ sowohl zu „einer verfahrensmäßigen Optimierung der Mitwirkung nationaler Parlamente an der innerstaatlichen Willensbildung“ in diesem Bereich als auch zu „einer innerstaatlichen Umsetzung der Subsidiari- 
tätskontrolle nationaler Parlamente“ (S. 461 f. und S. 469 - 472) erarbeitet; denn auf die Effizienz dieser innerstaatlichen Abläufe kommt es entscheidend an. Dass es an dieser Effizienz noch vieles zu verbessern gibt, hat schon die Untersuchung von Janowski in einem noch weiter gespannten Rahmen ergeben. Die von Mellein daraus gezogenen Folgerungen sind deshalb auch sehr beachtenswert.

Das gleicht aber nicht die Mängel aus, die aus den nicht hinreichend oder gar nicht gegebenen Antworten zu den Fragen einer direkten Einfügung nationaler parlamentarischer Willensbildung in die EU-Strukturen durch das Frühwarnsystem resultieren. Die Konfliktsituationen, die sich aus diesem System faktisch wie rechtlich ergeben, werden entweder gar nicht gesehen oder durch Euphorie über das Neue verdeckt. So sieht die Verfasserin die „direkte Einbeziehung der Parlamente im Anfangsstadium des Gesetzgebungsprozesses (als) eine neuartige ... Quelle demokratischer Legitimation“ an; denn es sei „ein Novum, dass dasjenige Organ, welches den Gesetzgebungsvorschlag vorlegt, der unmittelbaren Kontrolle aller mitgliedstaatlichen Parlamente unterliegt" (S.491). So weit, so gut - aber ist diese Neuartigkeit allein schon eine hinreichende Rechtfertigung für die durch sie heraufbeschworenen Vermischungsprobleme? Ohne jene Probleme auch nur annähernd hinreichend zu erörtern, konstatiert Mellein dazu schlicht, durch jene direkte Einbeziehung werde „die Beratung über Art, Sinn und Zweck der Gesetzgebung auf EU-Ebene ... verbessert, ohne dass die Entscheidungsfindung der Union wesentlich verzögert und das institutionelle Gleichgewicht gestört würden“" (S. 493). Nähere Erörterungen und Beweise für beides bleibt sie allerdings schuldig, und darin liegt der wesentliche Mangel des Buches.

Beide Untersuchungen sind zweifellos konkret für die noch ausstehende Praxis besonders nützlich. Der von Janowski gebührt aber der Vorrang, weil sie tiefer in die von der Einbindung nationaler Parlamente in die EU-Rechtsetzung heraufbeschworenen verfassungsrechtlichen Probleme eindringt, die von Mellein ignoriert oder zumindest verdeckt werden.

Uwe Leonardy

Der Kommissionspräsident der EU: Staatsoberhaupt, Regierungschef, „europäische Leitfigur"?

Staeglich, Simone: Der Kommissionspräsident als Oberhaupt der Europäischen Union. Vom primus inter pares zur europäischen Leitfigur, Duncker \& Humblot, Berlin 2007, 294 Seiten, $€ 72,-$.

Die Wahlen zum Europäischen Parlament zeichnen sich bisher nicht nur durch eine niedrige Wahlbeteiligung aus, sondern werden auch überwiegend mit Themen der nationalen Politik bestritten. In Deutschland gelten sie ähnlich wie Landtagswahlen als Stimmungsbarometer für die Unterstützung der Bundestagsparteien in der Bevölkerung. Als Gründe hierfür nennt man die fehlende Transparenz der Entscheidungswege in der EU und die fehlende Personalisierung der europäischen Politik. Während in den Mitgliedstaaten eine Wahl gleichzeitig als eine Entscheidung für oder gegen Tony Blair, Nicolas Sarkozy, Silvio Berlusconi oder Gerhard Schröder aufgefasst wird, fehlt der europäischen Politik das im Fernsehzeitalter so wichtige Gesicht. 